\title{
Green synthesis of silver nanoparticles by pulsed laser irradiation: effect of hydrophilicity of dispersing agents on size of particles
}

\author{
Umair Yaqub Qazi ${ }^{1}$, Zameer Shervani ${ }^{2 *}$ and Rahat Javaid ${ }^{3}$ \\ ${ }^{1}$ Division of Nanomaterials and Chemistry, Hefei National Laboratory for Physical Sciences at Microscale, University of Science and Technology of China, China \\ ${ }^{2}$ Division of Nanomaterials Production, Food and Energy Security Research and Product Centre, Japan \\ ${ }^{3}$ Fukushima Renewable Energy Institute, National Institute of Advanced Industrial Science and Technology, Japan
}

\begin{abstract}
Synthesis of homogeneous spherical silver nanoparticles ( $\mathrm{Ag}$ NPs) was conducted successfully by photochemical reduction of silver ions (Ag $\left.{ }^{+}\right)\left(10^{-4} \mathrm{M}\right)$ using near ultraviolet (UV) pulsed laser $(355 \mathrm{~nm})$ irradiation in an aqueous solution of surfactant sodium dodecyl sulphate (SDS) and cetyltrimethylammonium bromide (CTAB). Higher concentration of SDS and CTAB facilitated dispersion of reduced silver salt. Size of NPs was 72 and 163 nm in SDS and CTAB, respectively. Precursor silver acetate salt was irradiated for $30 \mathrm{~min}$. Effect of surfactant concentration, irradiation time, and hydrophilicity of surfactants were investigated. Amount of $\mathrm{Ag}^{+}$ions reduced increased with surfactant concentration and irradiation time.
\end{abstract}

Note: This article was retracted by the corresponding author from the journal "Advances in Nanoparticles" on an honest error and data still valid note. In this published article the electron generation by biphotonic mechanism has not been claimed as was done in the above retracted article.

\section{Introduction}

For last two decades synthesis of nono-metallic particles is gaining interest of researchers all over the world [1-4]. Favorably improved optical, electrical, magnetic, and pharmaceutical [5-7] properties of these nanocomposites make them potential candidates for catalysts, semiconductors, photo-voltic devices and medical applications for diagnosis and treatment [8,9] compared to bulk materials. In early period nanoparticles were synthesized in few milligram amount using toxic bulk organic solvents in inverted micelles for academic purposes [10]. In next step researchers were successful in synthesizing nanomaterials in large amount using severe conditions of high temperature and pressure [11]. Break through was obtained when Shervani and Yamamoto [12,13] discovered method of bulk nanoparticles production in glucose-water solution without addition of reducing agent at room temperature and pressure. Continuous synthesis of nanoparticles and particles adsorbed on charcoal surface were established for mass production in kilogram amount using a cheap micro-space continuous flow reactor, operated by ordinary pumps, technology under ambient conditions [14]. Catalytic activities of such nanoparticles were extraordinary high due large surface area and small size of particles when employed as catalysts. In top-down method where nanocrystals are removed from the substrate by high energy laser ablation is common [15-17]. Correard, et al. [18] prepared Au NPs by femtosecond laser ablation method in aqueous biocompatible solution of dextran and polyethylene glycol for medical applications. Authors have obtained blood red color Au NPs dispersed in water and had a prominent band at $520 \mathrm{~nm}$ in UV-vis spectrum. Size of NPs was in the range of 20-30 nm. In bottom up approach, Abid, et al. [19] prepared Ag NPs by reduction of $\mathrm{AgNO}_{3}$ in aqueous solution by high intense laser irradiation of pulse energy 12-14 mJ. Authors tested addition of 2-propanol in SDS solution and noticed that propanol addition decreases silver ions reduction. None of the authors investigated effect of surfactant hydrophilicity and anion or cationic nature of surfactant. In this article, we have described bottom up preparation of nanosized Ag NPs when silver salt exposed to intense nanosecond (ns) pulsed laser irradiation in SDS and CTAB aqueous solution. The effect of hydrophilicity of surfactants on NPs yield has been reported first. The method is important from green synthesis viewpoint since it does not involve addition of toxic reducing agents and yield and size of particles can be controlled by adjusting the laser irradiation time and type of surfactants used. The research is important from viewpoint of laser irradiation method application in continuous flow microspace reactors for production of nanomaterials on large scale in several grams in short time.

\section{Experimental}

\section{Materials}

Silver acetate of $99.0 \%$ purity was purchased from Sigma-Aldrich. Sodium dodecyl sulphate (SDS) and cetyl trimethyl ammonium bromide (CTAB) were from Wako Pure Chemicals, Co. Japan. Water used for samples' preparation was pure and deionized (resistivity, $18 \mathrm{M} \Omega^{-\mathrm{cm}}$ ).

\section{Preparation of silver nanoparticles}

To disperse reduced Ag NPs two kinds of stabilizing agents CTAB a cationic surfactant and SDS an anionic surfactant were used. In all experiments, a stock aqueous solution of silver acetate precursor salt of $0.01 \mathrm{M}$ was prepared in water. Salt concentration was kept at $10^{-4} \mathrm{M}$ or $5 \times 10^{-4} \mathrm{M}$. Concentration of stabilizing agents for CTAB was kept in

${ }^{\star}$ Correspondence to: Shervani Z, Food and Energy Security Research and Product Centre, Sendai, Japan, E-mail: shervani.nanotek@gmail.com

Key words: laser irradiation, pulsed laser, silver nanoparticles, photoreduction

Received: May 19, 2018; Accepted: May 31, 2018; Published: June 02, 2018 
the range $10^{-5}$ to $10^{-4} \mathrm{M}$ and for SDS was varied $10^{-4}$ to $10^{-3} \mathrm{M}$. Solutions of surfactant containing silver acetate precursor salt were transparent at experimental room temperature. For laser irradiation experiment, quartz cell $\left(10 \times 10 \times 45 \mathrm{~mm}^{3}\right)$ containing $3 \mathrm{~mL}$ of sample was used as a sample holder. Quartz cell was placed on a magnetic plate and solution was mixed by placing a magnetic bar and stirred at the rate 110 revolutions/min inside the cuvette to allow uniform irradiation of solution by laser beam. The laser beam was directly introduced to the sample solution without using focus lens. Figure 1 shows a schematic diagram of the experimental setup. Nanosecond near UV laser beam was generated by Quantel (Brilliant) manufacturer. The beam has specification of wavelength $355 \mathrm{~nm}$; pulse width $6 \mathrm{~ns}$; repetition rate: 10 $\mathrm{Hz}$ of energy $40 \mathrm{~mJ} /$ pulse maximum laser beam output. All experiments were performed at ambient conditions of room temperature and pressure.

\section{Characterization techniques}

The photochemical reduction of silver salt to silver metal was monitored visually by naked eyes and recording the UV-vis spectra of the preparations which are one of the most widely used technique for metallic nanomaterial characterization [20]. On exposer of Ag precursor salt solution to above pulsed laser, the colourless precursor solution changed to light yellow or dark yellow colour is the characteristic of Ag NPs formation.

Appearance of sharp plasmon resonance band at $400 \mathrm{~nm}$ confirmed presence of the of mono dispersed Ag NPs. UV-visible spectrophotometer Shimadzu UV-1600 was used for spectral recoding. Scanning Electron Microscopy (SEM) is well known technique to determine morphology and size of nanoparticles. SEM sample was prepared by drop casting a small portion of photo-reduced product on ITO substrates and dried in a desiccator. SEM images were recorded on Hitachi FE-SEM-S4300 apparatus operating at $20.0 \mathrm{kV}$ for structural and size determination of Ag NPs. The elemental analysis of Ag NPs were performed using an energy dispersive X-ray (EDX) spectrometer attached to the SEM machine. Dynamic laser light scattering (DLS) apparatus was employed to determine the size distribution of the particles. For size determination of synthesized Ag NPs, a high performance digital light scattering (DLS) particle size analyser Malvern Instrument Co., MAL501088 was used. DLS apparatus is known to measure the particles size including size of stabilizing agent enveloping the metallic particles along with the actual size of the metallic core.

\section{Results and Discussion}

\section{Optical properties of Ag NPs as observed by naked eyes}

The optical properties of metal NPs are highly dependent on their size and shape and have different colors due to the reflection of light [13]. After irradiating precursor salt solution containing surfactants SDS and CTAB with ns pulsed laser, a clear colorless solutions Figure 2a and Figure $3 \mathrm{a}$, respectively changed to light yellow color as shown

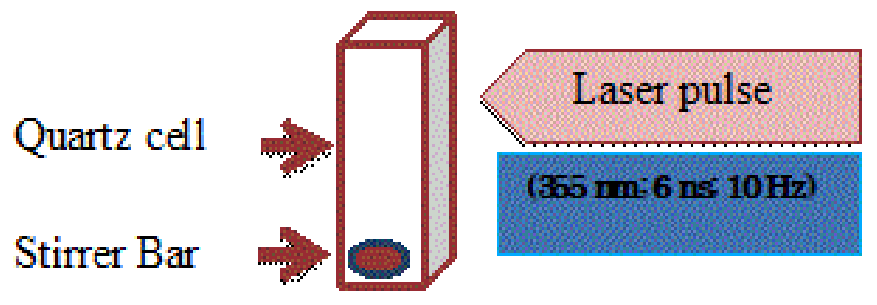

Figure 1. Experimental arrangement for ns pulsed laser fabrication of silver nanostructure in an aqueous solution of silver salt.

A B

C

D

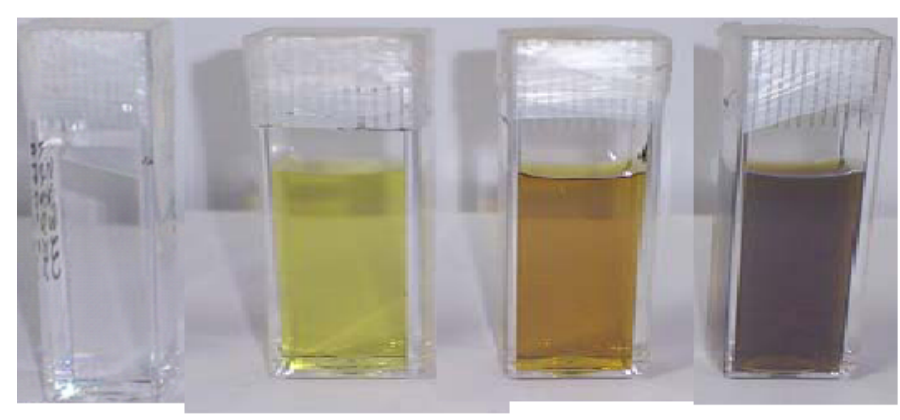

Figure 2. Digital optical images of phenomena showing the formation of Ag NPs when $5 \times 10^{-4} \mathrm{M} \mathrm{Ag}$ salts irradiated at different time in $10^{-3} \mathrm{M}$ SDS aqueous solution. A) zero irradiation time; B) 10 minutes; C) 15 minutes; D) 30 minutes.

A

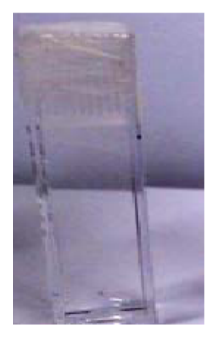

B

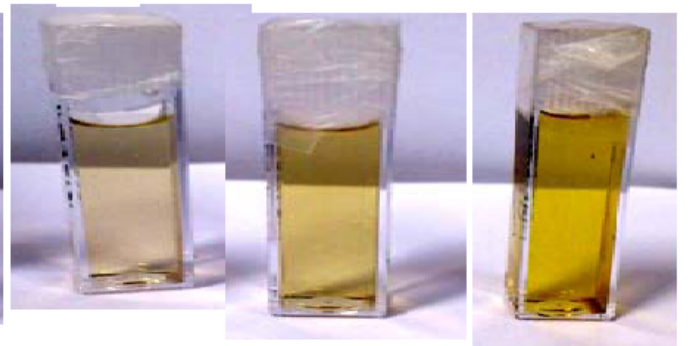

Figure 3. Digital optical images of phenomena showing the formation of Ag NPs when $10^{-4} \mathrm{M} \mathrm{Ag}$ salts irradiated ay different time in 10-4 M CTAB aqueous solution. A) zero irradiation time; B) 10 minutes; c) 15 minutes; D) 30 minutes.

in Figure $2 b$ and Figure $3 b$, respectively in just 10 min of irradiation time. The possibility of silver acetate $\left(\mathrm{Ag}^{+}\right)$reduction in CTAB solution by electron donor bromide ion has been ruled out by mixing sodium acetate with CTAB for 5, 10,15, and $30 \mathrm{~min}$. The solution did not change to yellow Ag NPs preparation. However, solution remained clear at these conditions. Thus NPs formation is really due to laser photon reduction not due to charge transfer from bromide ion. Tubes in Figures $2 \mathrm{~b}-2 \mathrm{~d}$ and Figures $3 \mathrm{~b}-3 \mathrm{~d}$ are the Ag NPs preparations for SDS and CTAB stabilised preparations, respectively, of increasing yield on 10, 15, and 30 min irradiation to ns laser as confirmed by increasing color intensity of the tubes. Increased NPs on longer irradiation was also confirmed by recording UV-vis spectra of preparations which will be discussed in the following section.

\section{UV-vis spectra recording of Ag NPs}

Figure 4 is UV-vis absorption spectra of colloidal solutions of Ag NPs obtained by laser irradiation in SDS aqueous solution of silver acetate $\left(5 \times 10^{-4} \mathrm{M}\right)$. Plasmon resonance band at $400 \mathrm{~nm}$ is the characteristics of mono dispersed Ag NPs. As the irradiation time increased from $3 \mathrm{~min}$ to 30 minutes, spectral intensity of the $400 \mathrm{~nm}$ increased to a maximum value of 3.25 and remained same even after exposer time increased beyond $30 \mathrm{~min}$. In $30 \mathrm{~min}$ of exposer time, all Ag+ ions $\left(5 \times 10^{-4} \mathrm{M}\right)$ were reduced by water electron. Amount of SDS needed to stabilize reduced Ag NPs was worked out by a separate experiment (Figure 5) in which increased amount of SDS was added in a tube containing $10^{-4} \mathrm{M}$ precursor salt. In varied SDS concentrations (8x10 ${ }^{4}, 5 \times 10^{-4}$, and $5 \times 10^{-3} \mathrm{M}$ ) salt was exposed to a fix $30 \mathrm{~min}$ ns laser. It was noticed that $5 \times 10^{-3} \mathrm{M}$ SDS was enough to stabilize Ag NPs. As the intensity of UV-vis Ag NPs band reached to a maximum of 3.25 (Figure 5). 


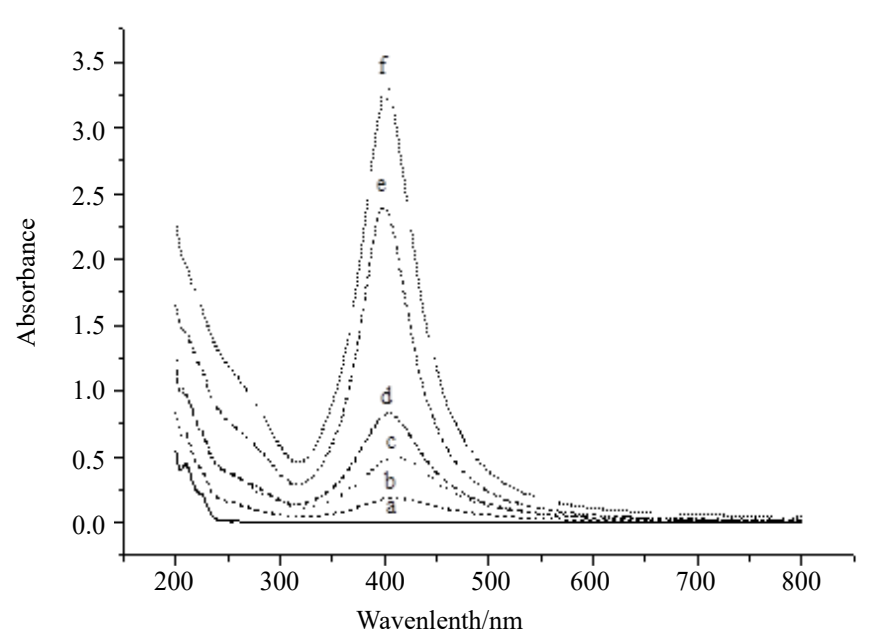

Figure 4. UV-vis spectral recording of Ag NPs formed by irradiating Ag salt using UV laser of $355 \mathrm{~nm}$ at different time at in SDS solution of $5 \times 10^{-1} \mathrm{M} \mathrm{Ag}$ salt concentration $5 \times$ $10^{-4} \mathrm{M}$. a) zero irradiation time; b) 3 min irradiation time; c) 5 minutes irradiation time; d) 10 minutes irradiation time; e) 15 minutes irradiation time; f) 30 minutes irradiation time.

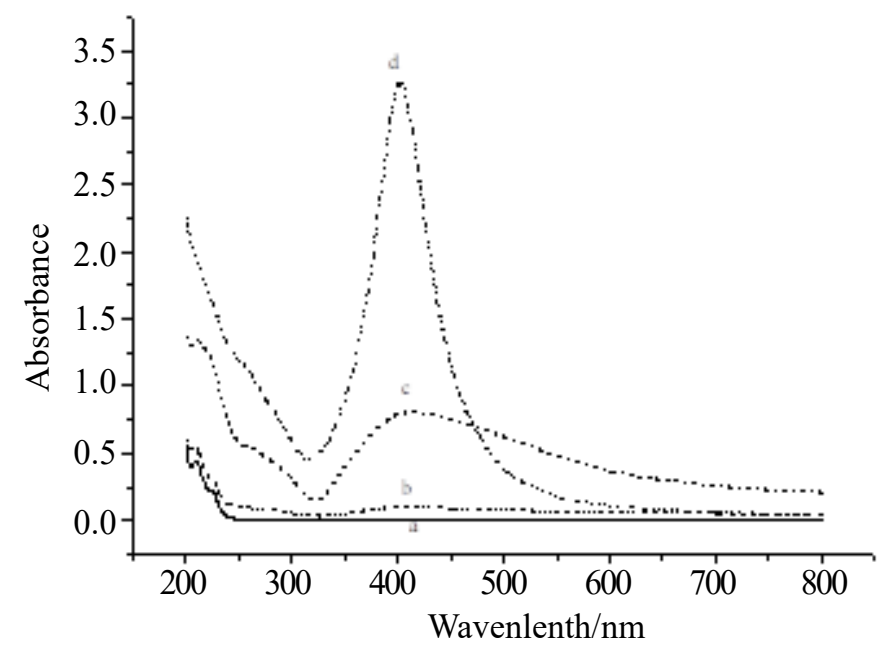

Figure 5. UV-vis spectral recording of Ag NPs formed by irradiating Ag salt using UV laser of $355 \mathrm{~nm}$ at different time at in SDS solution for 30 min irradiation time at different concentration of SDS. Ag salt concentration $10^{-4} \mathrm{M}$. a) zero irradiation time and SDS amount $8 \times 10^{-4} \mathrm{M}$; b) in absence of SDS; C) $5 \times 10^{-4} \mathrm{M}$; D) $5 \times 10^{-1} \mathrm{M}$.

In this research two types of surfactants an anionic SDS and a cationic CTAB were tested to stabilise Ag NPs in water solution. The experiment of CTAB is as follows. Figure 6 is the UV-vis recordings of neat $\mathrm{CTAB}, \mathrm{Ag}$ precursor salt, and salt admixed CTAB. When salt was added to CTAB, a shoulder in the spectrum was obtained at $250 \mathrm{~nm}$. Development of shoulder was attributed to the labial bromide anion which has capability to donate electron to reduce Ag cation while in anionic SDS negative charge on oxygen atoms is stable due presence of four oxygen atoms that limits the capability of SDS to donate or loose electron to reduce silver acetate. An experiment was conducted in a tube by mixing appropriate solution of silver acetate salt and CTAB, mixture became turbid after keeping solution for some time which was not found in SDS and silver acetate mixture. Thus, apparently CTAB bromide anion has tendency to reduce silver salt is the reason of appearance of shoulder in former. Thus, anionic and cationic surfactants behaved differently in preparation of Ag NPs. Moreover, SDS head group is more polar than CTAB thus dispersion power of
SDS is more than CTAB is revealed by 30 minutes irradiation UVvis spectral recording. In SDS absorbance of Ag NPs is 3.25 while it remained low (1.9) in CTAB.

Figure 7 is the spectra of Ag NPs when Ag salt was irradiated for 5, 10,15 , and $30 \mathrm{~min}$ in CTAB solution. Formation of Ag NPs enhanced on longer laser irradiation as was the case in SDS. Stabilization of reduced Ag NPs takes place even at low SDS concentration or pre-micellar or hemi-micellar concentration of $5 \times 10^{-4} \mathrm{M}$. Though SDS CMC is $8 \times 10-3$ $\mathrm{M}$, but hemi-micellar concentration is enough to capture the reduced $\mathrm{Ag}$ and stop them to aggregate. As shown in Figure 8, Ag NPs yield increased sharply in the premicellar domain of SDS as expected. After CMC NPs concentration did not increase so much. In this experiment amount of Ag salt $\left(5 \times 10^{-4} \mathrm{M}\right)$ and laser irradiation time $(30 \mathrm{~min})$ was kept constant. $\mathrm{Ag}^{+}$ions reduction occurred to $\mathrm{Ag}$ metal by the solvated electron formed by ns laser of $355 \mathrm{~nm}$ or $290 \mathrm{~mJ} / \mathrm{cm}^{2}$. As surfactant molecules are in solution, high energy non-metals are captured or shelled by surfactants to stop them aggregating in to large crystals.

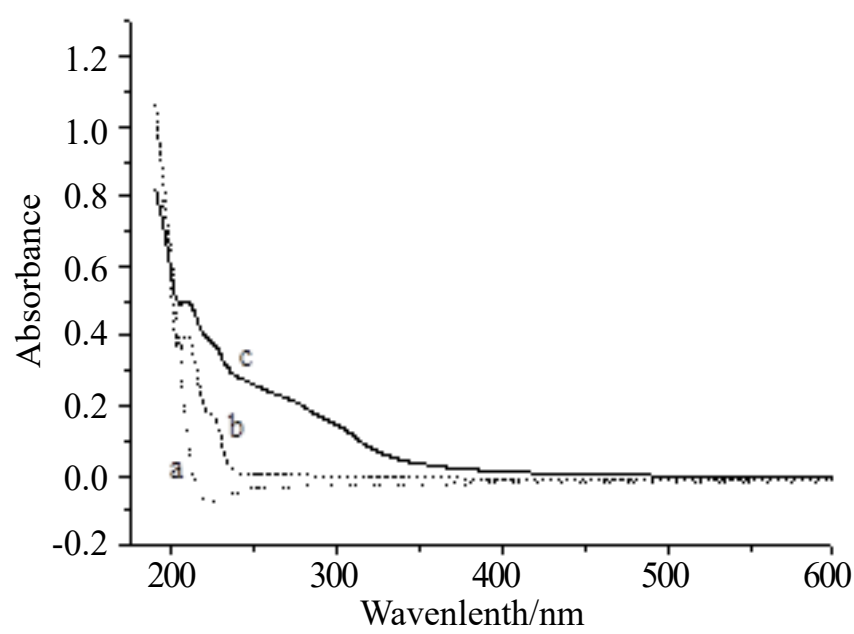

Figure 6. UV-vis spectral recording of a) CTAB $10^{-4} \mathrm{M}$; b) silver acetate salt; c) reduced silver in absence of any laser irradiation.

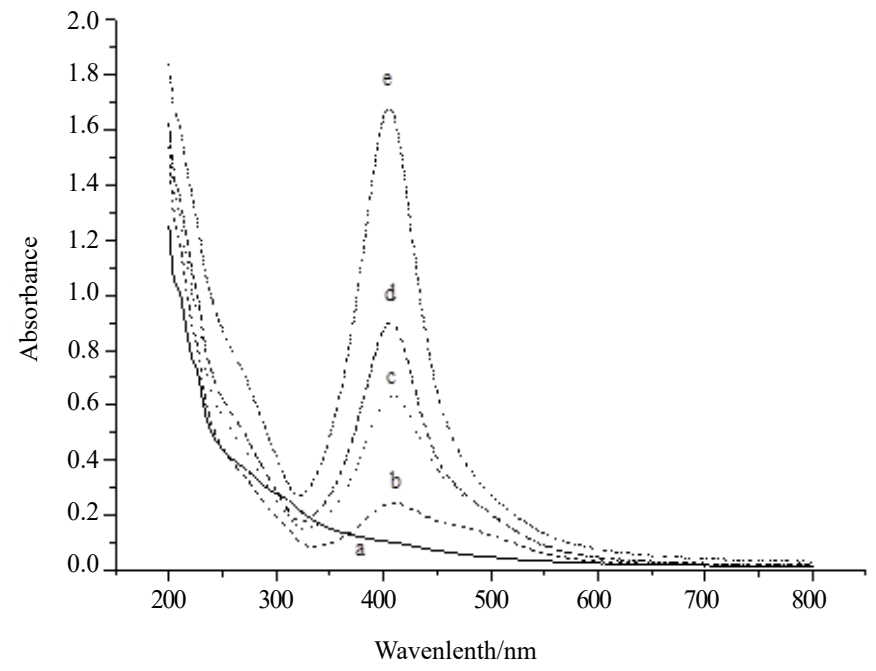

Figure 7. UV-vis spectral recording of Ag NPs obtained by irradiating Ag salt using UV laser of $355 \mathrm{~nm}$ at different time at in CTAB solution of $10^{-4} \mathrm{M} \mathrm{Ag}$ salt concentration $5 \times 10^{-4}$ M. a) zero irradiation time; b) 5 min irradiation time; c) 10 minutes irradiation time; d) 15 minutes irradiation time; e) 15 minutes irradiation time; f) 30 minutes irradiation time. 


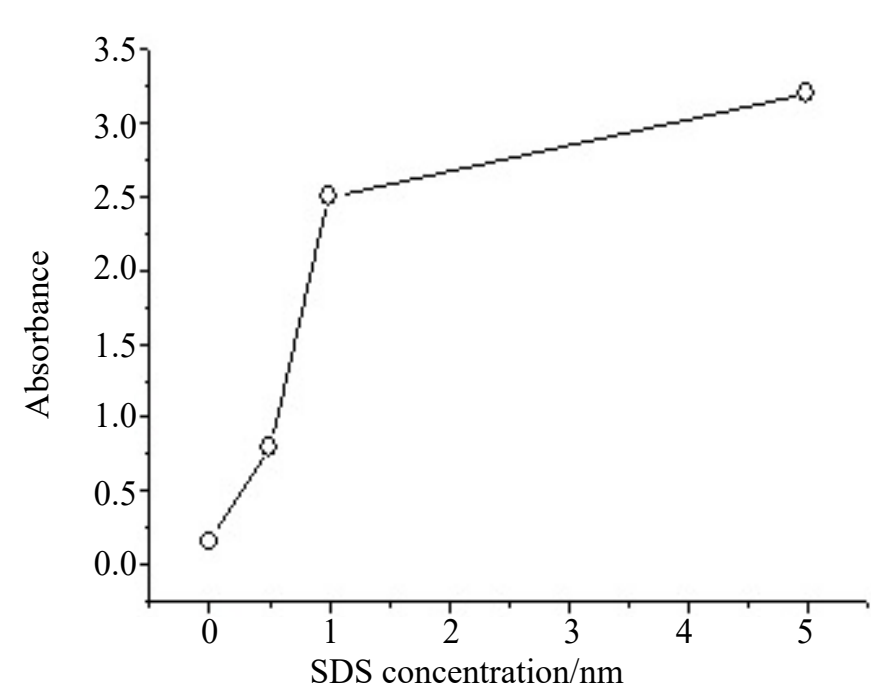

Figure 8. Change in photo-reduction efficiency vs SDS concentration measured at $400 \mathrm{~nm}$. $\mathrm{Ag}$ NPs dispersion enhanced with SD s concentration. Ag precursor salt is $5 \times 10^{-4} \mathrm{M}$.

\section{Morphology and size determination}

SEM and DLS measurements were conducted to determine size and polydispersity of Ag NPs. Samples were prepared by irradiating a $10^{-4} \mathrm{M} \mathrm{Ag}$ salt in $5 \times 10^{-3} \mathrm{M}$ SDS water solution for $30 \mathrm{~min}$ irradiation time. Figure 9 is the SEM image of Ag NPs recorded by loading sample on ITO holder. Particles are spherical shape and mono-dispersed with average particles' size of $72 \mathrm{~nm}$. Histogram bars of particles' size measured by DLS are shown in Figure 10 and particle distribution plot in Figure 11 when NPs were prepared by same preparation as in SEM recording. Ag NPs' size measured by SEM and DLS are same. Figure 12 is the SEM image of Ag NPs prepared by laser irradiation as defined in experimental section stabilized in CTAB surfactant. Compared to SDS, particle size is large in CTAB. It is $163 \mathrm{~nm}$ on the average. Particles distribution bars and graph are given in Figures 13 and 14, respectively. Most particles are in the range of 100 to 200 $\mathrm{nm}$. SDS is anionic surfactant with an immobile negative charge on oxygen atom moving over three other oxygen atoms. While CTAB is a cationic surfactant with a static negatively charged bromide ion capable of donating electron. As shown in Figure 6, there is no shoulder in silver acetate spectrum in water and $\mathrm{CTAB}$ solution when recorded separately. After mixing silver acetate and CTAB a prominent shoulder appeared without laser irradiation, this shoulder has been attributed to the formation of silver bromide or reduced silver salt where silver gets a negative charge from loosely held electron from bromide ions which is not the case in SDS surfactant.

\section{Composition of NPs}

Elemental analysis was performed by using EDX probe attached to the SEM apparatus. Figure 15 is EDX spectrum of Ag NPs prepared with ns laser irradiation method. The presence of a dominant band in the EDX spectrum at $3.0 \mathrm{keV}$ confirmed presence of silver metal. The results indicated that the reaction product was composed of high purity Ag element.

\section{Conclusion}

We demonstrated first the synthesis of stable Ag NPs from its salt using ns pulsed laser irradiation of $355 \mathrm{~nm}$. When high energy

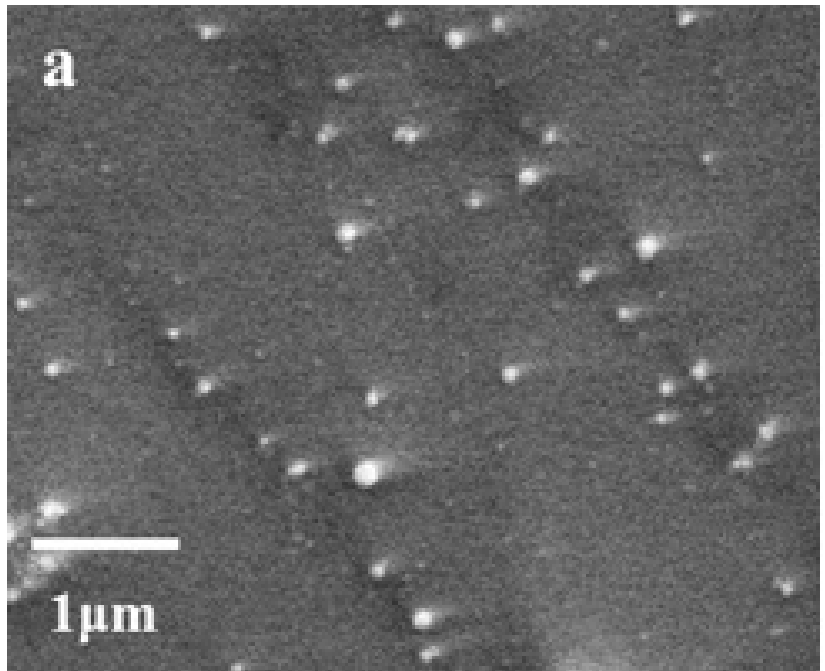

Figure 9. SEM image of Ag NPs of $10^{-4} \mathrm{M}$ silver salt irradiated for $30 \mathrm{~min}$ in SDS solution of $5 \times 10^{-3} \mathrm{M}$ solution. Monodispersed spherical particles of diameter 100-150 nm range are visible.

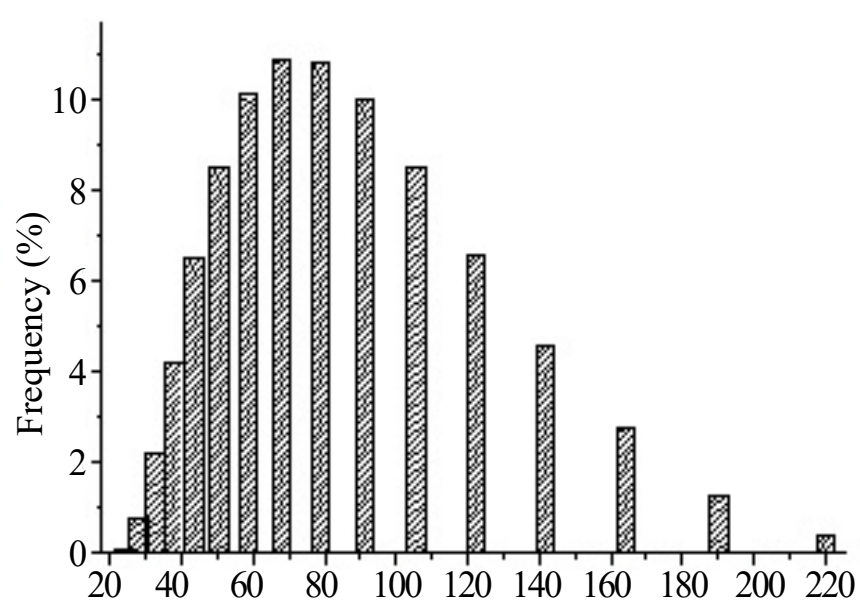

Figure 10. Ag NPs histogram obtained by irradiating salt for 30 mins as recorded by DLS SDS concentration $5 \times 10^{-3} \mathrm{M}$ and salt concentration $10^{-4} \mathrm{M}$.

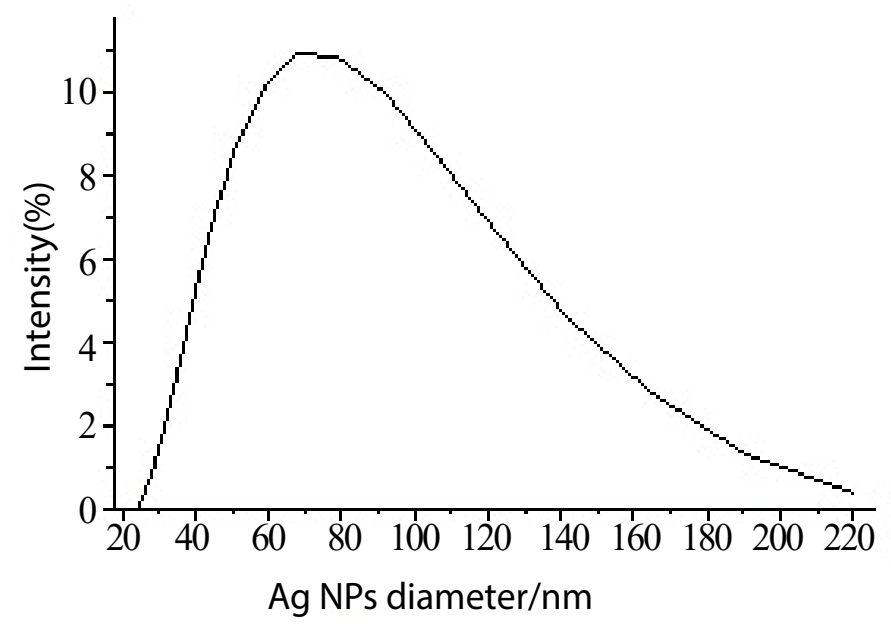

Figure 11. Ag NPs histogram obtained by irradiating salt for $30 \mathrm{~min}$ as recorded by DLS SDS concentration $5 \times 10^{-3} \mathrm{M}$ and salt concentration $10^{-4} \mathrm{M}$; average size $72.24 \mathrm{~nm}$. 


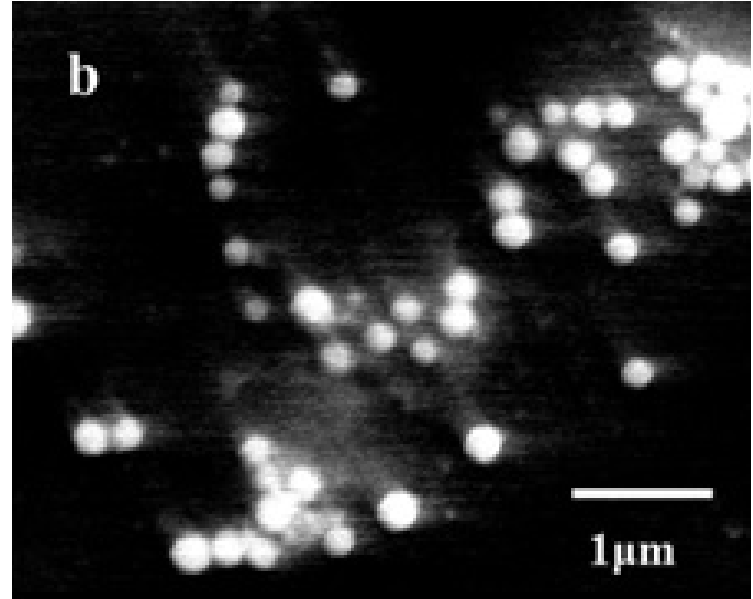

Figure 12. $\mathrm{SEM}$ ims $10^{-4} \mathrm{M}$ silver salt irradiated for $30 \mathrm{~min}$ in $\mathrm{CTAB}$ solution of $10^{-4} \mathrm{M}$ solution. Monodispersed spherical particles are visible.

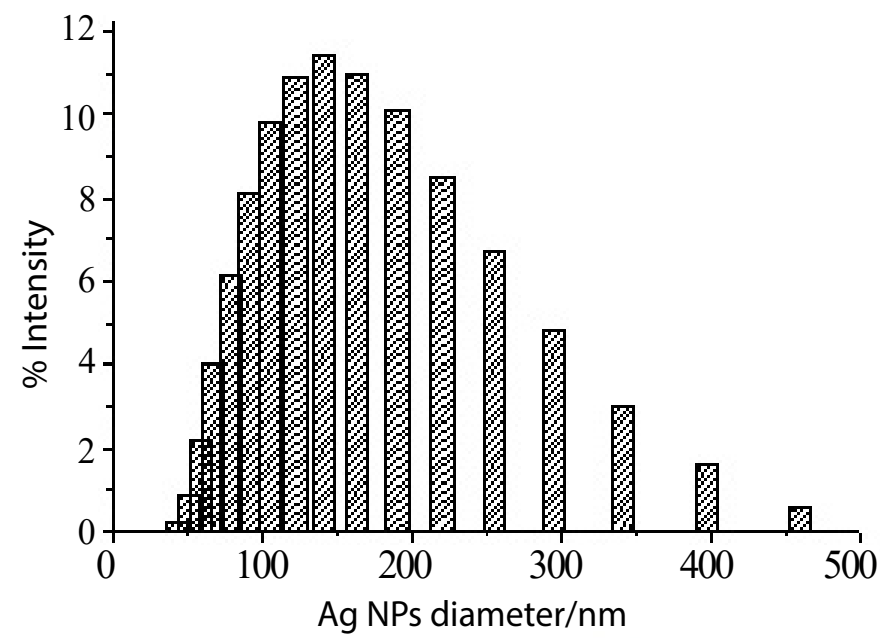

Figure 13. Ag NPs size distribution plot as measured by DLS and prepared by irradiating $\mathrm{Ag}$ salt in CTAB solution of concentration $10^{-4} \mathrm{M}$. Irradiation time is $30 \mathrm{~min}$. Concentration of $\mathrm{Ag}$ salt is $10^{-4} \mathrm{M}$.

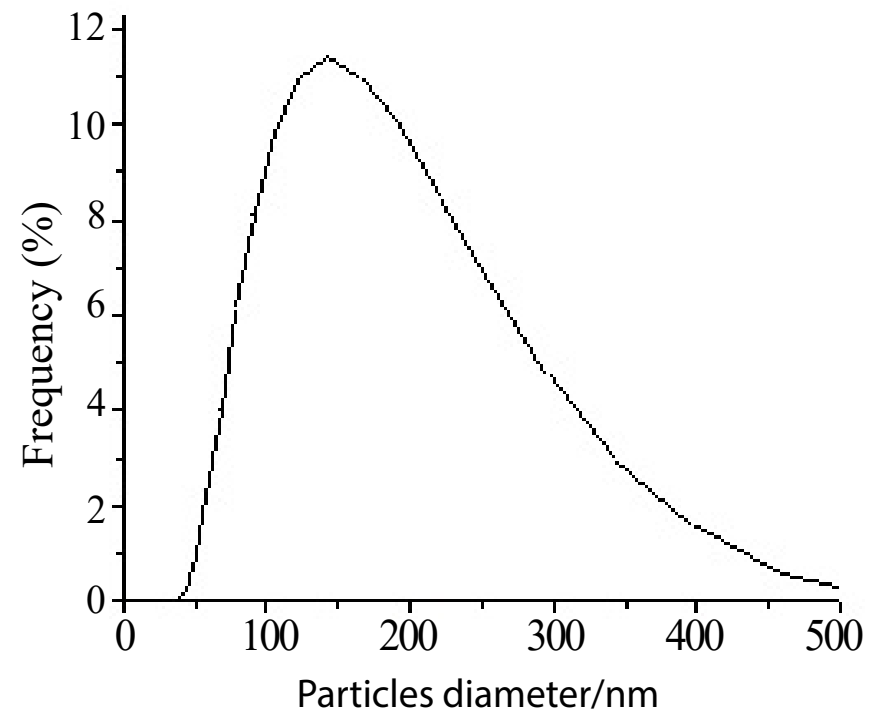

Figure 14. Ag NPs distribution plot obtained by irradiating salt for $30 \mathrm{~min}$ as recorded by SEM. SDS concentration $5 \times 10^{-3} \mathrm{M}$ and salt concentration $10^{-4} \mathrm{M}$.

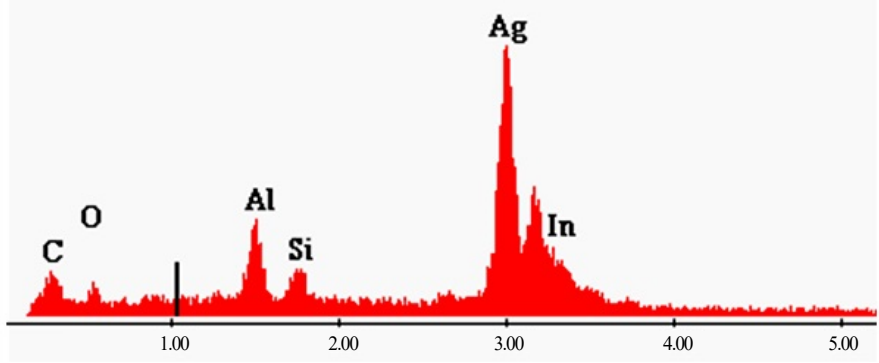

Figure 15. EDX spectrum of $\mathrm{Ag}$ NPs showing presence of $\mathrm{Ag}$ metal in preparation. Spectrum was recorded on ITO glass holder.

quanta of photons ejected electron from water which reduced silver cation. As soon as high energy nano-silver is formed they are stabilised by surfactants SDS or CTAB to form stable colloids. Size of particles were smaller $(72 \mathrm{~nm})$ in SDS compared to CTAB (163 $\mathrm{nm}$ ) due to more hydrophilic polar head group of SDS compared to hydrophobic CTAB head group. 30 min irradiation time was enough to reduce salt completely. Hemi-micellar concentration or pre-micellar concentration of both the surfactants is good to stabilized Ag NPs. EDX elemental analysis showed the silver composition of NPs. Method has given directionality to assemble continuous flow micro space reactor to produce nanomaterials in grams or kilograms amount in nontoxic solvent water for industrial and pharmaceutical applications.

\section{References}

1. Schmid G (1992) Large Clusters and Colloids, Metals in the Embryonic State Chemical Reviews 92: 1709-1727.

2. Pileni MP (1997) Nanosized Particles Made in Colloidal Assemblies. Langmuir 13: 3266-3276.

3. Henglein A (1989) Small-particle Research: Physicochemical Properties of Extremely Small Colloidal Metal and Semiconductor Particles. Chemical Reviews 89: 861-1873.

4. Linsebigler Al, Lu G, Yates JT (1995) Photocatalysis on $\mathrm{TiO}_{2}$ Surfaces: Principles, Mechanisms, and Selected Results. Chemical Reviews 95: 735-758.

5. Fox MA, Dulay MT (1993) Heterogeneous Photocatalysis. Chemical Reviews 93: $341-$ 357

6. Wang L, Gan X (2009) Antibody-functionalized Magnetic Manoparticles for Electrochemical Immunoassay of $\alpha-1$-fetoprotein in Human Serum. Microchimica Acta 164: 231-237.

7. Lince F, Marchisio DL, Barresi AA (2009) Smart Mixers and Reactors for the Production of Pharmaceutical Nanoparticles: Proof of Concept. Chemical Engineering Research and Design 87: 543-549.

8. Ming L (2003) Structure and Function of "Metalloantibiotics". Medical Research Reviews 23: 697-776.

9. Chuang TC, Liu YC, Wang CC (2005) Improved Surface-Enhanced Raman Scattering of Polypyrrole Electrodeposited on Roughened Substrates Composed of $\mathrm{Au}-\mathrm{Ag}$ Bimetallic Nanoparticles. Journal of Raman Spectroscopy 36: 704-708.

10. Pillai V, Kumar MJ, Hou P, Ayyub P, Shah DO (1995) Preparation of Nanoparticles of Silver Halides, SuperConductors and Magnetic Materials Using Water-in-Oil Microemulsions as Nano-reactors. Advances in Colloids and Interface Science 55: 241-269.

11. Adschiri T, Hakuta Y, Sue K, Arai K (2001) Hydrothermal Synthesis of Metal Oxide Nanoparticles at Supercritical Conditions. J Nanoparticles Res 3: 227-235.

12. Shervani Z, Yamamoto Y (2011) Size and Marphology Controlled Synthesis of Gold Nanoparticles in Green Solvent: Effect of Reducing Agents. Materials Letters 65: 92-95.

13. Shervani Z, Yamamoto Y (2011) Carbohydrate-Directed Synthesis of Silver and Gold Nanoparticles: Effect of the Structure of Carbohydrates and Reducing Agents on the Size and Morphology of the Composites. Carbohydrate Research 346: 651-658.

14. Nakamura T, Magara H, Herbani Y, Sato S (2011) Fabrication of Silver Nanoparticles by Highly Intense Laser Irrediation of Aqueous Solution. Applied Physiscs, A Materials Science \& Processing 104: 1021-1024. 
Qazi UY (2018) Green synthesis of silver nanoparticles by pulsed laser irradiation: effect of hydrophilicity of dispersing agents on size of particles

15. Abid JP, Wark AW, Brevet PF, Girault HH (2002) Preparation of Silver Nanoparticles in Solution from a silver salt by Laser Irradiation. Chemical Communications 792-793.

16. Sun YP, Atorngitjawat P, Meziani MJ (2001) Preparation of Silver Nanoparticles via Rapid Expansion of Water in Carbon Dioxide Microemulsion into Reductant Solution. Langmuir 17: 5707-5710.

17. Maciulevicius M, Vinciunas A, Brikas M, Butsen A, Tarasenka N, et al. (2013) Oneline Characterization of Gold Nanoparticles generated by Laser Ablation in Liquids. Physics Procedia 41: 531-538.
18. Ganjali M, Ganjali M, Vahdatkhah P, Marashi SMB (2015) Synthesis of Ni Nanoparticles by Pulsed Laser Ablation Method in Liquid Phase. Procedia Materials Science 11: 359-363.

19. Amendola V, Polizzi S, Meneghetti M, (2006) Laser Ablation Synthesis of Gold Nanoparticles in Organic Solvents. J Phys Chem B 110: 7232-7237. [Crossref]

20. Correard F, Maximova K, Esteve M, Villard C, Roy M, et al. (2014) Gold Nanoparticles Prepared by Laser Ablation in Aqueous Biocompatible Solutions: Assessment of Safety and Biological Identity for Nanomedicine Applications. Int $J$ Nanomedicine 9 : 5415-5430. [Crossref]

Copyright: $@ 2018$ Qazi UY. This is an open-access article distributed under the terms of the Creative Commons Attribution License, which permits unrestricted use, distribution, and reproduction in any medium, provided the original author and source are credited. 